\title{
Robust Discrete-time Consensus of Multi-agent Systems with Uncertain Interaction
}

\author{
Dongkun Han, Graziano Chesi
}

\begin{abstract}
This paper addresses robust discrete-time consensus problem of multiple agents with uncertain structure, where the network coupling weights are supposed polynomial functions of an uncertain vector constrained in a semialgebraic set. Based on the Lyapunov stability theory, a necessary and sufficient condition for robust discrete-time consensus is proposed. Then, we investigate the robust discrete-time consensus with positive weighted network, and a necessary and sufficient condition is also provided based on the property of an uncertain matrix. Corresponding sufficient conditions for robust discrete-time consensus are derived by solving a linear matrix inequality (LMI) problem built by exploiting sum-of-squares (SOS) polynomials. Some examples illustrate the proposed results.
\end{abstract}

\section{INTRODUCTION}

Recent years have witnessed an increasing number of researches on cooperative control, in particular significant efforts have been made to study consensus problems of multi-agent systems. Most of relevant researches focus on the multi-agent systems with fixed topology, and corresponding consensus protocols have been extensively investigated. It has been shown that for system with first-order dynamics, consensus can be achieved if and only if the associated network has a directed spanning tree, while for system with second-order dynamics, not only is consensus determined by the topology, but also the Laplacian matrix whose real and imaginary components of the eigenvalues contribute to reaching consensus [1], [2].

Besides the fixed topology, in order to make a more realistic multi-agent model, a growing number of attentions have been paid to the system with random network, uncertain perturbations, misbehaving agents and unmeasurable inputs [3][5]. [6] exploits nonlinear couplings among a nonidentical, nonlinear network with continuous-time dynamics described by Euler-Lagrange equations. [7] investigates a periodically time-varying topology where agent obtains its position and velocity states only at sampling time. Another type of switching topology, the stochastic switching topology driven by a finite ergodic Markov chain, is proposed and studied in [8]. The robust consensus of multi-agent systems with diverse input delays is studied in [9], which assumes there is asymmetric linear perturbations of coupling weights in communication network, while [10] supposes a linear perturbation in input control affecting velocity states. In order to address constraints of communication, [11] employs the proximity graph to model the limited range of interaction channel. Numerous though kinds of transforming structures of multi-agent systems are,

Authors are both with the Department of Electrical and Electronic Engineering, University of Hong Kong, Pokfulam Road, Hong Kong (email:dkhan@eee.hku.hk) none research has been interested on the robust discretetime consensus where the coupling weights of network are disturbed by polynomial functions of uncertain parameters.

This paper investigates robust discrete-time consensus problems of multi-agent network with uncertain parameters constrained in a given set. Specifically, the coupling weights of network are assumed to be polynomial functions of an uncertain vector, which is constrained in a semialgebraic set. Firstly, a necessary and sufficient condition is provided for robust discrete-time consensus by exploiting Lyapunov stability theory. And then, for the same problem, we derive a sufficient condition by solving a linear matrix inequality (LMI) problem built by using SOS matrix polynomials (see e.g. [12] about LMIs and [13] and references therein about SOS polynomials). In addition, based on analysis of the eigenvalues of an uncertain matrix, this paper proposes a necessary and sufficient condition for robust discrete-time consensus with positive weighted network. Based on this condition, a sufficient condition for robust discrete-time consensus is derived by solving an LMI problem built by exploiting SOS polynomials. Some numerical examples illustrate the proposed results.

The paper is organized as follows. Section II provides the problem formulation and some preliminaries about graph theory and SOS polynomials. In Section III, the conditions for robust discrete-time consensus are provided. In Section IV, some numerical examples are reported. Lastly, Section V concludes the paper with some final remarks.

\section{PRELIMINARIES}

\section{A. Problem Formulation}

The notation used throughout the paper is as follows:

- $\mathbb{N}, \mathbb{R}$ : natural and real number sets;

- $A^{\prime}:$ transpose of $A$;

- $A>0(A \geq 0)$ : symmetric positive definite (semidefinite) matrix $A$;

- $0_{n}$ : origin of $\mathbb{R}^{n}$;

- $1_{n}=(1, \ldots, 1)^{\prime} \mathbb{R}^{n}$;

- $I_{n}: n \times n$ identity matrix;

- $\operatorname{img}(A)$ : image of matrix $A$;

- $\operatorname{ker}(A)$ : null space of matrix $A$;

- $A \otimes B$ : Kronecker product of matrices $A$ and $B$;

- $\operatorname{spc}(A)$ : set of eigenvalues of $\mathrm{A} \in \mathbb{R}^{n \times n}$, i.e.

$$
\operatorname{spc}(A)=\left\{\lambda \in \mathbb{C}: \operatorname{det}\left(\lambda I_{n}-A\right)=0\right\} .
$$

According to graph theory, a weighted and directed graph $\mathscr{G}$ can be described by three elements, i.e. $\mathscr{G}=(\mathscr{A}, \mathscr{E}, G)$, where $\mathscr{A}=\left\{A_{1}, \ldots, A_{n}\right\}$ is a finite, nonempty set to describe the set 
of $n$ nodes of a multi-agent system, $\mathscr{E}$ is the set of directed edges belonging to $\mathscr{A} \times \mathscr{A}$ and $G$ is a weighted adjacency matrix displayed by $G=\left(G_{i j}\right)_{n \times n}$. A directed edge from $A_{j}$ to $A_{i}$ is defined by $G_{i j}$ and it represents an information transmitting channel from the $j$-th node to the $i$-th node, where vehicle $A_{j}$ and vehicle $A_{i}$ are called parent node and child node respectively. In this paper, a directed graph is investigated to model the network of multi-agent systems.

A directed path from $A_{i}$ to $A_{j}$ is defined as a sequence of directed edges $\left(A_{i}, A_{i 1}\right),\left(A_{i 1}, A_{i 2}\right), \ldots,\left(A_{i l}, A_{j}\right)$ in directed network with distinct nodes $A_{i k}, k=1, \ldots, l$. A graph $\mathscr{G}$ is called a strongly connected graph if there is a directed path between any pair of distinct notes $A_{i}$ and $A_{j}$. If a node $i$ has the property that, for any node $j$ different from $i$, there is a directed path from $i$ to $j$, we define node $i$ as a root. A directed tree is a directed graph where exactly one root exists and except the root, every node in $\mathscr{G}$ has exactly one parent. A spanning tree of a directed graph is a directed tree in which graph edges connect all the nodes of the graph. We say a graph has a spanning tree if any subset of edges involves or forms a spanning tree [14].

Uncertain multi-agent systems are considered in this paper. For robust first-order consensus, the discrete-time consensus scheme for a network with $n$ agents can be expressed by

$$
x_{i}(k+1)=\frac{1}{\sum_{j=1}^{n} G_{i j}(\theta)} \sum_{j=1}^{n} G_{i j}(\theta) x_{j}(k), \quad i=1, \ldots, n
$$

where $x_{i} \in \mathbb{R}$ is the state of the $i$-th node, $\theta \in \mathbb{R}^{r}$ is an uncertain vector, and $G_{i j}(\theta): \mathbb{R}^{r} \rightarrow \mathbb{R}$ is the $(i, j)$-th entry of the uncertain weighted adjacency matrix $G(\theta)=\left(G_{i j}(\theta)\right)_{n \times n}$. The uncertain vector $\theta$ is constrained in a set as $\theta \in \Omega$ where

$$
\Omega=\left\{\theta \in \mathbb{R}^{r}: s_{i}(\theta) \geq 0 \forall i=1, \ldots, h\right\}
$$

for some $s_{1}, \ldots, s_{h}: \mathbb{R}^{r} \rightarrow \mathbb{R}$. In the sequel we will assume that $G_{i j}(\theta)$ and $s_{1}(\theta), \ldots, s_{h}(\theta)$ are polynomials. Moreover, we say that $G(\theta)$ is positive if $G_{i j}(\theta)>0$ for all $i, j$ and for all $\theta \in \Omega$, otherwise $G(\theta)$ is called non-positive.

Based on the multi-agent system defined above, we propose the problems of robust discrete-time consensus.

Problem 1. To establish if, for any initial state, the uncertain multi-agent system with scheme (1) achieves consensus, i.e.

$$
\begin{aligned}
& \lim _{k \rightarrow \infty} x_{i}(k)-x_{j}(k)=0 \\
& \forall \theta \in \Omega, \forall x_{i}(0) \in \mathbb{R}, \forall i, j \in\{1,2, \ldots, n\} .
\end{aligned}
$$

Problem 2. To establish if, for any initial state, the uncertain multi-agent system achieves consensus with scheme (1) and with positive weighted network, i.e.

$$
\begin{aligned}
& \lim _{k \rightarrow \infty} x_{i}(k)-x_{j}(k)=0 \quad G_{i j}(\theta) \geq 0 \\
& \forall \theta \in \Omega, \forall x_{i}(0) \in \mathbb{R}, \forall i, j \in\{1,2, \ldots, n\} .
\end{aligned}
$$

In order to address these problems, we rewrite (1) as

$$
x(k+1)=D(\theta) x(k)
$$

where $x=\left(x_{1}, \ldots, x_{n}\right)^{\prime}$ is the state vector, and $D(\theta)=$ $\left(D_{i j}(\theta)\right)_{n \times n}$ is an uncertain matrix given by

$$
D_{i j}(\theta)=\frac{G_{i j}(\theta)}{\sum_{j=1}^{n} G_{i j}(\theta)} .
$$

It is worth pointing out that the uncertain matrix $D(\theta)$ has the property that

$$
\sum_{j=1}^{n} D_{i j}(\theta)=1 \quad \forall i=1, \ldots, n .
$$

\section{B. SOS Polynomials}

A powerful tool for establishing whether a (multivariate) polynomial is nonnegative consists of establishing whether it is a SOS polynomial via an LMI feasibility test.

Specifically, let $f(x)$ be a polynomial of degree $2 m$ in $x \in$ $\mathbb{R}^{r}$. Then, $f(x)$ can be written as

$$
f(x)=x^{\{m\}^{\prime}}(F+C(\delta)) x^{\{m\}}
$$

where $x^{\{m\}} \in \mathbb{R}^{\sigma(r, m)}$ is a vector containing all monomials of degree less than or equal to $m$ in $x$, and the length of $x^{\{m\}}$ is expressed as

$$
\sigma(r, m)=\frac{(r+m) !}{r ! m !},
$$

$F \in \mathbb{R}^{\sigma(r, m) \times \sigma(r, m)}$ is a symmetric matrix, and $C(\delta)$ is a linear parametrization of the linear subspace

$$
\mathcal{C}=\left\{C=C^{\prime}: x^{\{m\}^{\prime}} C x^{\{m\}}=0\right\} .
$$

The representation (8) is known as Gram matrix method [15] and square matrix representation (SMR) [16]. This representation was introduced in [16] for establishing whether a polynomial is SOS via LMIs. Indeed, $f(x)$ is SOS if there exist polynomials $f_{1}(x), f_{2}(x), \ldots$ such that

$$
f(x)=\sum_{i} f_{i}(x)^{2}
$$

and this condition holds if and only if there exists $\delta$ such that the following LMI feasibility test holds:

$$
F+C(\delta) \geq 0
$$

This techniques can also be used in the case of matrix polynomials. Specifically, let $M(x)$ be a symmetric matrix polynomial of size $s \times s$ of degree $2 m$ in $x \in \mathbb{R}^{r}$ (this means that all the entries of $M(x)$ are polynomials of degree $2 m$ in $x)$. Then, $M(\theta)$ can be written as

$$
M(\theta)=\Delta(\bar{M}+E(\delta), m, s)
$$

where

$\Delta(\bar{M}+E(\delta), m, s)=\left(\theta^{\{m\}} \otimes I_{s}\right)^{\prime}(\bar{M}+E(\delta))\left(\theta^{\{m\}} \otimes I_{s}\right)$,

$\bar{M} \in \mathbb{R}^{s \sigma(r, m) \times s \sigma(r, m)}$ is a symmetric matrix, and $E(\delta)$ is a linear parametrization of the linear subspace

$$
\mathcal{E}=\left\{E=E^{\prime}:\left(x^{\{m\}} \otimes I_{s}\right)^{\prime} E\left(x^{\{m\}} \otimes I_{s}\right)=0\right\} .
$$


The representation (14) allows one to establish whether a matrix polynomial is SOS via LMIs. Indeed, $M(x)$ is SOS if there exist matrix polynomials $M_{1}(x), M_{2}(x), \ldots$ such that

$$
M(x)=\sum_{i} M_{i}(x)^{\prime} M_{i}(x)
$$

and this condition holds if and only if there exists $\delta$ such that the following LMI feasibility test holds:

$$
\bar{M}+E(\delta) \geq 0 \text {. }
$$

It is worth mentioning that SOS polynomials have been exploited in optimization over polynomials since a long time, in particular [17] has been one of the pioneering works on this topic. For details, the reader can also refer to the book [18] and the survey [13].

\section{CONDitions FOR Robust CONSEnsus}

In this section we derive the proposed conditions for robust discrete-time consensus.

\section{A. Robust Consensus of General Network}

Lyapunov stability theory is of great significance to study the property of dynamical system. For the first time, we associate the robust discrete-time consensus with Lyapunov stability theory, and we provide a new condition for investigating robust first-order consensus based on matrix inequalities. Specifically, define a matrix $V \in \mathbb{R}^{n \times n-1}$ such that

$$
\operatorname{img}(V)=\operatorname{ker}\left(1_{n}^{\prime}\right), V^{\prime} V=I_{n-1} .
$$

Then we can transform the uncertain matrix $D(\theta)$ as:

$$
\widehat{D}(\theta)=V^{\prime} D(\theta) V .
$$

By exploiting the discrete-time Lyapunov inequality, we can obtain the following condition to check both Problem 1 and Problem 2.

Theorem 1: Robust discrete-time consensus for uncertain multi-agent system (with both positive and non-positive weighted network) can be achieved if and only if there exists a symmetric function $P: \mathbb{R}^{r} \rightarrow \mathbb{R}^{n-1 \times n-1}$ such that

$$
\left\{\begin{array}{l}
P(\theta)>0 \\
P(\theta)-\widehat{D}(\theta)^{\prime} P(\theta) \widehat{D}(\theta)>0 \quad \forall \theta \in \Omega .
\end{array}\right.
$$

Proof Observe $1_{n}$ is an eigenvector of $D(\theta)$ corresponding to the eigenvalue 1, and from (18) one can obtain that $V^{\prime} D(\theta) V$ has the same eigenvalues of $D(\theta)$ except that the algebraic multiplicity of the eigenvalue 1 has been decreased of one. Based on the condition that eigenvalue 1 is a simple eigenvalue of $D(\theta)$, one gets that

$$
\operatorname{spc}(\widehat{D}(\theta)) \cup\{1\}=\operatorname{spc}(D(\theta)) .
$$

Let us define a dynamical system

$$
\hat{x}(k+1)=\widehat{D}(\theta) \hat{x}(k) .
$$

Observe that $\forall \gamma \in \mathbb{R}, \hat{x}_{0}=\gamma 1_{n-1}$ is the equilibrium point of (22), hence the statement that robust discrete-time consensus can be achieved is equivalent to the statement that (22) is asymptotically stable. According to (21) and the Lyapunov stability theorem, (22) is asymptotically stable $\forall \theta \in \Omega$ if and only if $D(\theta)$ has exactly one simple eigenvalue 1 and all the spectral norms of the other eigenvalues are smaller than 1 . From Lyapunov stability theorem for linear systems, this is equivalent to say that there exists $P(\theta)$ such that (20) holds $\forall \theta \in \Omega$. This completes the proof.

In order to investigate the condition of Theorem 1, we can exploit SOS matrix polynomials. Observe elements of $D(\theta)$ may be rational functions in $\theta$, hence we firstly make a transformation on $D(\theta)$ as follows

$$
\widetilde{D}(\theta)=\zeta(\theta) D(\theta)
$$

where the $\zeta(\theta)=\operatorname{LCM}\left(\sum_{j=1}^{n} G_{i j}(\theta)\right), \forall i=1,2, \ldots, n$, and LCM is short for the Least Common Multiplier. Then we can get a matrix polynomial $\widetilde{D}(\theta)$ whose elements are polynomial functions of $\theta$. Accordingly, the conditions of Theorem 1 are changed to

$$
\left\{\begin{array}{l}
P(\theta)>0 \\
\zeta(\theta)^{2} P(\theta)-\widehat{\widetilde{D}}(\theta)^{\prime} P(\theta) \widehat{\widetilde{D}}(\theta)>0
\end{array} \quad \forall \theta \in \Omega .\right.
$$

where

$$
\widehat{\widetilde{D}}(\theta)=V^{\prime} \widetilde{D}(\theta) V
$$

Specifically, let $P(\theta)$ and $H_{i}(\theta), i=1, \ldots, h$, be symmetric matrix polynomials, and $s_{i}$ is described in (2). Then define

$$
R(\theta)=\zeta(\theta)^{2} P(\theta)-\widehat{\widetilde{D}}(\theta)^{\prime} P(\theta) \widehat{\widetilde{D}}(\theta)-\sum_{i=1}^{h} H_{i}(\theta) s_{i}(\theta)
$$

We can verify that (20) holds if there exists $c>0$ such that

$$
\left\{\begin{array}{l}
H_{i}(\theta) \text { is SOS } \\
P(\theta)-I_{n-1} \text { is SOS } \\
R(\theta)-c I_{n-1} \text { is SOS. }
\end{array}\right.
$$

By exploiting the representation of matrix polynomials introduced in Section II, the condition (27) can be established by a convex optimization problem with LMI constraints. Specifically, let $2 m_{i}, 2 m$ and $2 m_{0}$ be the degree of $H_{i}(\theta), P(\theta)-I$, and $R(\theta)-c I$ respectively. We define the representations as follows:

$$
\begin{aligned}
H_{i}(\theta) & =\Delta\left(\bar{H}_{i}, m_{i}, n-1\right) \\
H_{i}(\theta) s_{i}(\theta) & =\Delta\left(\bar{U}_{i}\left(\bar{H}_{i}\right), m_{0}, n-1\right) \\
P(\theta) & =\Delta(\bar{P}, m, n-1) \\
R(\theta) & =\Delta\left(\bar{M}+E(\delta), m_{0}, n-1\right)
\end{aligned}
$$

where $\bar{H}_{i}, \bar{U}_{i}\left(\bar{H}_{i}\right), \bar{P}, \bar{M}$ and $E(\delta)$ are symmetric matrices. Then we have the following result.

Corollary 1: With both positive and non-positive weighted network, the robust discrete-time consensus can be achieved if one gets $c^{*}>0$ by solving following convex optimization 
problem with LMI constraints.

$$
\begin{gathered}
\qquad c^{*}=\sup _{c, \bar{H}_{i}, \bar{P}, \delta} c \\
\text { s.t. }\left\{\begin{array}{l}
\bar{H}_{i} \geq 0 \\
\bar{P} \geq I_{d_{1}} \\
\bar{M}+E(\delta)-c I_{d_{2}}-\sum_{i=1}^{h} \bar{U}_{i}\left(\bar{H}_{i}\right) \geq 0
\end{array}\right.
\end{gathered}
$$

where $d_{1}$ and $d_{2}$ are the sizes of $\bar{P}$ and $\bar{M}$, respectively.

Proof Since $\bar{H}_{i} \geq 0$, from (28) one can obtain that $H_{i}(\theta) \geq 0$. Similarly, one gets $P(\theta) \geq 0, R(\theta) \geq 0$. In addition, it is given that $s_{i}(\theta)>0$ and

$$
\left(\theta^{\left\{m_{0}\right\}} \otimes I_{n-1}\right)^{\prime} E(\delta)\left(\theta^{\left\{m_{0}\right\}} \otimes I_{n-1}\right)=0 \forall \theta \in \mathbb{R}^{r} .
$$

Accordingly, from $c^{*}>0$ in (29) one can obtain that $\forall \theta \in \Omega$, there exists $c>0$ with $H_{i}(\theta) \geq 0, P(\theta)>0$ and

$$
\begin{aligned}
0 \leq & \zeta(\theta)^{2} P(\theta)-\widehat{\widetilde{D}}(\theta)^{\prime} P(\theta) \widehat{\widetilde{D}}(\theta)-\sum_{i=1}^{h} H_{i}(\theta) s_{i}(\theta) \\
& -c I_{n-1} \\
\leq & \zeta(\theta)^{2} P(\theta)-\widehat{\widetilde{D}}(\theta)^{\prime} P(\theta) \widehat{\widetilde{D}}(\theta)-c I_{n-1} \\
< & \zeta(\theta)^{2} P(\theta)-\widehat{\widetilde{D}}(\theta)^{\prime} P(\theta) \widehat{\widetilde{D}}(\theta) .
\end{aligned}
$$

Then it directly follows that (24) and (20) hold. The proof is thus completed.

Remark 1: Corollary 1 provides a sufficient condition for robust consensus with both positive and non-positive weighted network. The conservatism of this approach is determined by the length of uncertain vector $\theta$ and the degree of $H_{i}(\theta), P(\theta)$ and $R(\theta)$. To reduce the conservative standard of Corollary 1 is firmly associated with the Hilbert's 17th problem which concerns on the gap between SOS polynomials and positive polynomials [18].

\section{B. Robust Consensus of positive weighted network}

In this subsection, we investigate the robust consensus conditions for uncertain systems with positive weighted network, It has been found that, for graph $\mathscr{G}=(\mathscr{A}, \mathscr{E}, G)$, the consensus of the directed network is determined by the topology of network. First of all, based on the condition given in [19] for the case of multi-agent systems without uncertainty, let us introduce the following preliminary result, which extends to the case of uncertain multi-agent systems.

Lemma 1: For a given uncertain matrix $D(\theta)$ in (6) and an uncertain network with $G_{i j}(\theta) \geq 0$, the following three statements are equivalent.

a) Robust discrete-time consensus can be achieved.

b) $\forall \theta \in \Omega, D(\theta)$ has exactly one simple eigenvalue 1 and all the other eigenvalues satisfy $|\lambda|<1$.

c) $\forall \theta \in \Omega$, the directed graph $\mathscr{G}$ has a spanning tree.

Proof Assume the uncertain matrix $D(\theta)$ is constructed by (6). Then, the three statements are equivalent and follow directly from the analogous ones found for the case of multi-agent systems without uncertainty, refering to the Corollary 2.18, Lemma 2.19 and Theorem 2.20 of [19].

From Lemma 1 we know that the eigenvalue distribution of $D(\theta)$ determines whether the robust discrete-time consensus can be achieved. For a positive weighted network, if we can find a way to establish the condition of statement b) in Lemma 1 , the robust consentability of the positive weighted network can be obtained directly. Based on this lemma we propose a necessary and sufficient condition for robust discrete-time consensus with positive weighted network.

Theorem 2: For a given uncertain matrix $D(\theta)$ in (6) and a positive weighted network, i.e. $\exists e_{i j} \in \mathscr{E}$ if and only if $G_{i j}(\theta) \geq 0$, the robust discrete-time consensus can be achieved if and only if

$$
q(\theta) \neq 0, \forall \theta \in \Omega
$$

where

$$
q(\theta)=\left.\left(\frac{d}{d \lambda} l(\lambda, \theta)\right)\right|_{\lambda=1}
$$

and

$$
l(\lambda, \theta)=\operatorname{det}(\lambda I-D(\theta)) .
$$

Proof Since $G_{i j}(\theta)>0$, by (6) one obtains $D_{i j}(\theta)>0$, $\forall i, j=1,2, \ldots, n$. Observe that $D(\theta)$ has the same positive constant row sums given by 1 , then 1 is an eigenvalue of $D(\theta)$ with an associated eigenvector $1_{n}$ and the spectral radius of $D(\theta)$ is 1 , which follows directly from the properties of nonnegative matrices [20]. Thus, for all non-one eigenvalue, we have that $\left|\lambda_{i}(D(\theta))\right|<1, \forall i=1,2 \ldots, n, \forall \theta \in \Omega$. Moreover, condition (31) implies that $D(\theta)$ has exactly one simple eigenvalue $1, \forall \theta \in \Omega$. Therefore, statement $\mathrm{b}$ ) in Lemma 1 and condition (31) are equivalent. This completes the proof.

Remark 2: As well we can use Theorem 1 to check the robust consensus of positive weighted network. Nevertheless Theorem 2 is advanced in terms of numerical complexity, for the reason that a symmetric parameter-dependent function $P(\theta)$ should be found under condition (20) which is an convex optimization problem, while for condition (31), it is numerically simple to calculate the roots of a polynomial function and check whether these roots are in the constrained set defined by (2).

In order to check the condition of Theorem 2, we can also utilize the SOS polynomials which amounts to solving an LMI problem. Since $q(\theta)$ may be a rational function, we first transform $l(\lambda, \theta)$ and $q(\theta)$ as:

$$
\begin{aligned}
\tilde{l}(\lambda, \theta) & =\zeta(\theta)^{n} \operatorname{det}\left(\lambda I_{n}-D(\theta)\right) \\
\tilde{q}(\theta) & =\left.\left(\frac{d}{d \lambda} \tilde{l}(\lambda, \theta)\right)\right|_{\lambda=1} .
\end{aligned}
$$


Then we can obtain following optimization problem:

$$
\begin{gathered}
c^{*}=\sup _{c, g_{i}(\theta)} c \\
\text { s.t. }\left\{\begin{array}{l}
g_{i}(\theta) \text { is SOS } \\
(-1)^{k} \tilde{q}(\theta)-c-\sum_{i=1}^{h} g_{i}(\theta) s_{i}(\theta) \text { is SOS }
\end{array}\right.
\end{gathered}
$$

where $k \in\{0,1\}$ is defined as

$$
k= \begin{cases}0 & \text { if } \tilde{q}\left(\theta_{0}\right)>0 \\ 1 & \text { otherwise }\end{cases}
$$

where $\theta_{0}$ is any vector $\theta$ freely chosen in $\Omega$. Then, condition of Theorem 2 holds if $c^{*}>0$.

Actually, we can calculate $c^{*}$ in (35) by solving an LMI problem via using the technique of representation of polynomials. Specifically, let $2 m_{i}, 2 m_{0}$ be the degree of $g_{i}(\theta)$ and $(-1)^{k} \tilde{q}(\theta)-c-\sum_{i=1}^{h} g_{i}(\theta) s_{i}(\theta)$ respectively. Let us define the representations

$$
\begin{aligned}
g_{i}(\theta) & =\theta^{\left\{m_{i}\right\}^{\prime}} J_{i} \theta^{\left\{m_{i}\right\}} \\
g_{i}(\theta) s_{i}(\theta) & =\theta^{\left\{m_{0}\right\}^{\prime}} Q_{i}\left(J_{i}\right) \theta^{\left\{m_{0}\right\}} \\
(-1)^{k} \tilde{q}(\theta) & =\theta^{\left\{m_{0}\right\}^{\prime}}(F+C(\delta)) \theta^{\left\{m_{0}\right\}} \\
1 & =\theta^{\left\{m_{0}\right\}^{\prime}} W \theta^{\left\{m_{0}\right\}}
\end{aligned}
$$

where $J_{i}, Q_{i}\left(J_{i}\right), F, C(\delta)$ and $W$ are symmetric matrices. Then we have the following result.

Corollary 2: With a positive weighted network, the robust discrete-time consensus can be achieved if one gets $c^{*}>0$ by solving a convex optimization problem as follows

$$
\begin{gathered}
c^{*}=\sup _{c, J_{i}, \delta} c \\
\text { s.t. }\left\{\begin{array}{l}
J_{i} \geq 0 \\
F+C(\delta)-c W-\sum_{i=1}^{h} Q_{i}\left(J_{i}\right) \geq 0 .
\end{array}\right.
\end{gathered}
$$

Proof The derivation process is same to the corresponding part of Corollary 1. $\forall \theta \in \Omega$, whenever $c^{*}>0$ and the constraints in (35) hold, one can get $c>0$ with $g_{i}(\theta) \geq 0$ and

$$
\begin{aligned}
0 & \leq(-1)^{k} \tilde{q}(\theta)-c-\sum_{i=1}^{h} g_{i}(\theta) s_{i}(\theta) \\
& \leq(-1)^{k} \tilde{q}(\theta)-c,
\end{aligned}
$$

i.e. $c$ is a lower bound of $(-1)^{k} \tilde{q}(\theta), \forall \theta \in \Omega$. Then it directly follows that (31) holds. This completes the proof.

\section{NUMERICAL EXAMPLES}

In this section, a couple of examples are provided to illustrate the proposed approach. These optimization problems are solved with standard MATLAB toolboxes SeDuMi and SMRSOFT [21], [22].

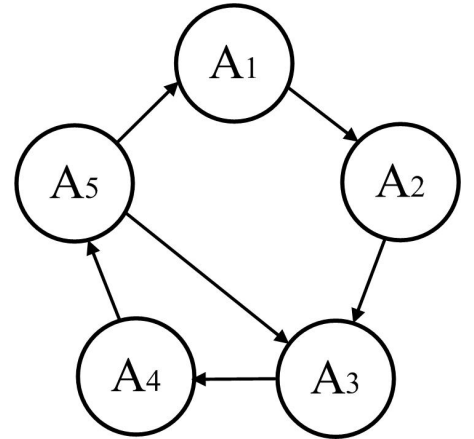

Fig. 1. Topology of Five-agent system.

\section{A. Example 1}

In this example, a five-agent system is considered as Figure 1. This network is perturbed by an uncertain parameter $\theta$. Thus, we have $n=5$ and $r=1$. Specifically the weighted adjacency matrix is given by

$$
G(\theta)=\left[\begin{array}{ccccc}
1 & 0 & 0 & 0 & 1 \\
2 & 1 & 0 & 0 & 0 \\
0 & 2-2 \theta & 1 & 0 & 1 \\
0 & 0 & 2 & 1 & 0 \\
0 & 0 & 0 & 1-\theta & 1
\end{array}\right]
$$

where $\theta$ is constrained in a selected set $\Omega$ as

$$
\Omega=[-1,1] .
$$

Here we describe $\Omega$ as a polynomial inequality in (2) with

$$
s_{1}(\theta)=1-\theta^{2} .
$$

By using (6), we can obtain the uncertain matrix $D(\theta)$ as:

$$
D(\theta)=\left[\begin{array}{ccccc}
\frac{1}{2} & 0 & 0 & 0 & \frac{1}{2} \\
\frac{2}{3} & \frac{1}{3} & 0 & 0 & 0 \\
0 & \frac{2-2 \theta}{4-2 \theta} & \frac{1}{4-2 \theta} & 0 & \frac{1}{4-2 \theta} \\
0 & 0 & \frac{2}{3} & \frac{1}{3} & 0 \\
0 & 0 & 0 & \frac{1-\theta}{2-\theta} & \frac{1}{2-\theta}
\end{array}\right] .
$$

Thus, we find $\zeta(\theta)$ as:

$$
\zeta(\theta)=12-6 \theta .
$$

First, let us apply Theorem (1) to establish whether robust consensus can be achieved with Figure 1. According to (20), we choose a constant matrix function $P(\theta)$ to guarantee the positivity in this case. By solving the optimization problem (29), we can find $c^{*}=+\infty$, i.e. (27) holds with any positive scalar $c$. Then the robust discrete-time consensus can be achieved.

Next, we find that all the entries of the weighted adjacency matrix $G(\theta)$ are non-negative for all $\theta \in \Omega$, thus the Theorem 2 can either be utilized to investigate the robust discrete-time consensus. Specifically, the polynomial $\tilde{q}(\theta)$ in (34) can be calculated as:

$$
\begin{array}{r}
\tilde{q}(\theta)=-12096 \theta^{5}+102384 \theta^{4}-342576 \theta^{3} \\
+565920 \theta^{2}-461376 \theta+148608 .
\end{array}
$$


According to Theorem 2 and (34), robust discrete-time consensus is achieved if and only if $\tilde{q}(\theta) \neq 0$ for all $\theta \in \Omega$. In addition, we also find that $\tilde{q}(\theta)$ is an univariate polynomial whose roots are 2, 2, 2, $1.2321+0.1324 \mathrm{i}$ and $1.2321-0.1324 \mathrm{i}$, the absolute values of which are all located outside $[-1,1]$. Hence we obtain $\tilde{q}(\theta) \neq 0$ for all $\theta \in \Omega$. Meanwhile, we calculate the quantity $c^{*}$ in (38). By selecting multiplier $g_{1}(\theta)$ of degree 2 , we have that $k=0$, and finally we find $c^{*}=864$, which proves that condition (31) in Theorem 2 is satisfied.

Furthermore, we investigate the eigenvalues of $D(\theta)$ and the topology of network to further validate the proposed theory. From Figure 1, obviously the network has a directed spanning tree, i.e. $\left\{\left(A_{1}, A_{2}\right),\left(A_{2}, A_{3}\right),\left(A_{3}, A_{4}\right),\left(A_{4}, A_{5}\right)\right\}$. In addition, for all $\theta$ in (40), $D(\theta)$ has exactly one simple eigenvalue 1 , and the spectral radius of $\widehat{D}$ is 0.7586 . Hence the uncertain network can reach robust discrete-time consensus, whose conclusion definitely coincides with ours.

\section{B. Example 2}

With the same topology shown in Figure 1, another uncertain five-agent system is considered in this case. Different from the first example, it is assumed that the network is affected by two uncertain parameters, i.e. $\theta_{1}$ and $\theta_{2}$. Meanwhile the network has both positive and non-positive weighting factors. Specifically the uncertain weighted adjacency matrix $G(\theta)$ is assumed to be

$$
\left[\begin{array}{ccccc}
1 & 0 & 0 & 0 & 1 \\
2 \theta_{1}+7 & 1 & 0 & 0 & 0 \\
0 & \theta_{2}-\theta_{1} & 1 & 0 & 3+\theta_{1} \\
0 & 0 & \theta_{1}+3 & 1 & 0 \\
0 & 0 & 0 & 0.5 \theta_{2}+1 & 1
\end{array}\right]
$$

where $\theta \in \mathbb{R}^{2}$ is constrained in the set $\Omega$ chosen as

$$
\Omega=\{\theta:\|\theta\| \leq 1\} .
$$

Hence, we have $n=5$ and $r=2$. Moreover, $\Omega$ can be described as in (2) with

$$
s_{1}(\theta)=1-\theta_{1}^{2}-\theta_{2}^{2} .
$$

And according to the uncertain matrix $D(\theta)$ calculated by (6), we can obtain $\zeta(\theta)$ as:

$$
\zeta(\theta)=32+8 \theta_{1}+8 \theta_{2}+2 \theta_{1} \theta_{2} .
$$

In this case, $G(\theta)$ may not be positive, hence let us apply condition (20) to investigate robust discrete-time consensus. First we find a constant matrix function $P(\theta)$ satisfying (20), and then we get $c^{*}=+\infty$ by solving (29). That means the robust discrete-time consensus is achieved.

\section{CONCLUSIONS}

In this paper we have addressed the problems of establishing robust discrete-time consensus for uncertain multiagent systems. And for these problems we have provided necessary and sufficient conditions based on the Lyapunov stability theory and the eigenvalue analysis of uncertain matrix $D(\theta)$. In addition, we have derived sufficient conditions that amount to solving LMI problems built by exploiting SOS matrix polynomials. Now special efforts are made to reduce the methdological conservatism.

\section{ACKNOWLEDGMENT}

The authors would like to thank the Editors and the Reviewers for their valuable suggestions and comments.

\section{REFERENCES}

[1] W. Yu, G. Chen, and M. Cao. Some necessary and sufficient conditions for second-order consensus in multi-agent dynamical systems. Automatica, 46(6):1089-1095, 2010.

[2] W. Ren and R.W. Beard. Consensus seeking in multiagent systems under dynamically changing interaction topologies. IEEE Trans. Autom. Control, 50(5):655-661, 2005.

[3] Y. Gao and L. Wang. A necessary and sufficient condition for consensus of continuous-time agents over undirected time-varying networks. IEEE Trans. Autom. Control, 56(5):1226-1231, 2011.

[4] P. Lin and Y. Jia. Further results on decentralised coordination in networks of agents with second-order dynamics. Control Theory and Application IET, 3(6):957-970, 2009.

[5] N. Abaid and M. Porfiri. Consensus over numerosity-constrained random network. IEEE Trans. Autom. Control, 56(3):649-654, 2011.

[6] E. Nuño, R. Ortega, L. Basañez, and D. Hill. Synchronization of networks of nonidentical euler-lagrange sytems with uncertain parameters and communication delays. IEEE Trans. Autom. Control, 56(4):935941, 2011.

[7] Y. Gao and L. Wang. Sampled-data based consensus of continuous-time multi-agent systems with time-varying topology. IEEE Trans. Autom. Control, 56(5):1226-1231, 2011.

[8] Y. Zhang and Y. Tian. Consentability and protocol design of multi-agent systems with stochastic switching topology. Automatica, 45:1195-1201, 2009.

[9] Y. Tian and C. Liu. Robust consensus of multi-agent systems with diverse input delays and asymmetric interconnection perturbations. $A u$ tomatica, 45:1347-1353, 2009.

[10] W. Zhang, Z. Zheng, and Y. Guo. Robust consensus for uncertain multiagent systems on directed communication topologies. In in Proc. IEEE Conf. Decision and Control, pages 6317-6322, Atlanta, GA, 2010.

[11] U. Münz, A. Papachristodoulou, and F. Allgöwer. Robust consensus controller design for nonlinear relative degree two multi-agent systems with communication constraints. IEEE Trans. Autom. Control, 56(1):145151,2011

[12] S. Boyd, L. El Ghaoui, E. Feron, and V. Balakrishnan. Linear Matrix Inequalities in System and Control Theory, volume 15 of Studies in Applied Mathematics. SIAM, Philadelphia, PA, June 1994.

[13] G. Chesi. LMI techniques for optimization over polynomials in control: a survey. IEEE Trans. on Automatic Control, 55(11):2500-2510, 2010.

[14] C. Godsil and G. Royle. Algebraic Graph Theory. Springer-Verlag, 2001.

[15] M. Choi, T. Lam, and B. Reznick. Sums of squares of real polynomials. In Symposia in Pure Mathematics, pages 103-126, 1995.

[16] G. Chesi, A. Tesi, A. Vicino, and R. Genesio. On convexification of some minimum distance problems. In European Control Conf. Karlsruhe, Germany, 1999.

[17] N. Z. Shor. Class of global minimum bounds of polynomial functions (in Russian). Cybernetics, 23:731-734, 1987.

[18] G. Chesi, A. Garulli, A. Tesi, and A. Vicino. Homogeneous Polynomial Forms for Robustness Analysis of Uncertain Systems. Springer-Verlag, 2009.

[19] W. Ren and R. Beard. Distributed Consensus in Multi-vehicle Cooperative Control. Communications and Control Engineering. SpringerVerlag, 2008.

[20] R.A. Horn and C.R. Johnson. Matrix Analysis. Cambridge Univ. Press, 1985.

[21] J. Sturm. Using SeDuMi 1.02, a MATLAB toolbox for optimization over symmetric cones. optim. Meth. Software, 11-12:625-653, 1999.

[22] G. Chesi. SMRSOFT: A Matlab toolbox for optimization over polynomials and dynamical systems study via SOS programming. Available at http://www.eee.hku.hk/ chesi, 2011. 